\title{
On strong law for blockwise $M$-orthogonal random fields
}

\author{
Xu Wu-ling and Wang Zhong-zhi*
}

${ }^{\text {*Correspondence: }}$ zhongzhiw@126.com

School of Mathematics \& Physics, AnHui University of Technology, Ma'anshan, 243002, People's Republic of China

\begin{abstract}
We consider M-orthogonal random fields. Using a lemma from summability theory, we prove strong law of large numbers for blockwise $M$-orthogonal random fields under various moment conditions, thereby generalizing some results in the literature from independent random fields.
\end{abstract}

MSC: $60 \mathrm{~F} 15$

Keywords: random fields; limit theorem; M-orthogonal

\section{Introduction}

Recently, Móricz et al. (cf. [1]) using the summability theory proved a strong law of large numbers for blockwise $M$-dependent random variables under moment conditions. Huan and Quang ( $c f$. [2]) established the Doob's inequality for martingale difference arrays and provided a sufficient condition, so that the strong law of large numbers would hold for an arbitrary array of random elements without imposing any geometric condition on the $\mathrm{Ba}$ nach space. Quang et al. (cf. [3]) provided conditions to obtain the almost sure convergence for a double array of blockwise $M$-dependent random elements $\left\{V_{m n}: m \geq 1, n \geq 1\right\}$, taking values in a real separable Rademacher-type $p(1<p \leq 2)$, and they also demonstrated that some of the well-known theorems in the literature were special cases of their results.

Let $\mathbb{Z}_{+}^{d}$, where $d$ is a positive integer, denote the positive integer $d$-dimensional lattice points. Motivated by the results above, in this paper, we are going to study strong law of large numbers for $M$-orthogonal random fields $\left(X_{\mathbf{n}}\right)$ with $\mathbf{n} \in \mathbb{Z}_{+}^{d}$. The notation $\mathbf{m} \prec \mathbf{n}$, where $\mathbf{m}=\left(m_{1}, m_{2}, \ldots, m_{d}\right)$ and $\mathbf{n}=\left(n_{1}, n_{2}, \ldots, n_{d}\right)$, means that $m_{i} \leq n_{i}, 1 \leq i \leq d, \mathbf{n} \rightarrow \infty$ means $n_{1} \wedge n_{2} \wedge \cdots \wedge n_{d} \rightarrow \infty$.

Definition 1 The sequence $\left\{X_{\mathbf{n}}, \mathbf{n} \in \mathbb{Z}_{+}^{d}\right\}$ is called a sequence of $M$-orthogonal random variables if

$$
E X_{\mathbf{k}} X_{\mathbf{l}}=0
$$

for all $\mathbf{k}$ and $\mathbf{l}$ with $\max _{1 \leq i \leq d}\left|k_{i}-l_{i}\right|>m$.

A somewhat weaker dependence condition is given by the following definition.

Definition 2 For given sequences of natural numbers $\left(\beta_{k}^{i}\right),\left(\beta_{k}^{i}\right) \uparrow \infty(1 \leq i \leq d)$ (as $k \rightarrow \infty)$, we say $\left(X_{\mathbf{n}}\right)$ is blockwise $M$-orthogonal with respect to blocks $\left[\beta_{k_{1}}^{(1)}, \beta_{k_{1}+1}^{(1)}\right) \times$ 
$\left[\beta_{k_{2}}^{(2)}, \beta_{k_{2}+1}^{(2)}\right) \times \cdots \times\left[\beta_{k_{d}}^{(d)}, \beta_{k_{d}+1}^{(d)}\right)$ if for all $k_{i}(1 \leq i \leq d) \in \mathbb{N}$, the random variables $\left(X_{\mathbf{n}}\right)$ are $M$-orthogonal for indices $\mathbf{n} \in\left[\beta_{k_{1}}^{(1)}, \beta_{k_{1}+1}^{(1)}\right) \times\left[\beta_{k_{2}}^{(2)}, \beta_{k_{2}+1}^{(2)}\right) \times \cdots \times\left[\beta_{k_{d}}^{(d)}, \beta_{k_{d}+1}^{(d)}\right.$ ).

The latter definition is a generalization of the corresponding definition for the onedimensional case. It allows the random variables in the different blocks be strongly dependent. The particular case $\beta_{k_{i}}^{(i)}=k^{\alpha}, \beta_{k_{i}+1}^{(i)}=(k+1)^{\alpha}, \alpha>1, k \in \mathbb{N}(1 \leq i \leq d)$ is especially interesting.

In order to prove our main results, we shall state the following two lemmas, and it will be shown that they play a key role in the proof.

Lemma 1 (cf. [4]) Let $\left\{X_{\mathbf{n}}, \mathbf{n} \in \mathbb{Z}_{+}^{d}\right\}$ be a random field with $M$-orthogonal, centered random variables, if $E X_{\mathbf{n}}^{2}<\infty$ for all $\mathbf{n} \in \mathbb{Z}_{+}^{d}$, then we have

$$
E\left(\max _{\mathbf{k}<\mathbf{n}}\left|S_{\mathbf{k}}\right|\right)^{2} \leq(m+1)^{d} \cdot\left(\prod_{i=1}^{d}\left(\log _{2} 2 n_{i}\right)^{2}\right) \cdot \sum_{\mathbf{k}<\mathbf{n}} E X_{\mathbf{k}}^{2} .
$$

Next, we consider sequences $\left(S_{\mathbf{n}}\right)=\left(S_{\mathbf{n}}\right)_{\mathbf{n}=(1,1, \ldots, 1)}^{\infty}$ of real or complex numbers. We say that $\left(S_{\mathbf{n}}\right)$ is boundedly convergent to $S$ if $\sup _{\mathbf{n} \succ(1,1, \ldots, 1)}\left|S_{\mathbf{n}}\right|<\infty$ and if for any $\varepsilon>0$ there exists some $n_{0}(\varepsilon)$ such that $\left|S_{\mathbf{n}}-S\right|<\varepsilon$ for all $n_{i} \geq n_{0}(\varepsilon)(1 \leq i \leq d)$ (Pringsheim convergence). We write shortly $S_{\mathbf{n}} \rightarrow S$ (bd).

Lemma $2(c f .[5])$ Let $\Psi_{1}(\cdot), \Psi_{2}(\cdot), \ldots, \Psi_{d}(\cdot)$ be positive, strictly increasing unbounded functions on $[0, \infty)$, and let $\left(k_{n}^{(i)}\right)_{0}^{\infty}, 1 \leq i \leq d$ be strictly increasing sequences of integers with $k_{0}^{(i)}=0,1 \leq i \leq d$. Consider the following relations for array sequences $\left(S_{\mathbf{n}}\right)$ as $\mathbf{n} \rightarrow \infty$ :

$$
\begin{aligned}
t_{\mathbf{n}}:= & \frac{1}{\left(\Psi_{1}\left(k_{n_{1}+1}^{(1)}\right)-\Psi_{1}\left(k_{n_{1}}^{(1)}\right)\right) \cdots\left(\Psi_{d}\left(k_{n_{d}+1}^{(d)}\right)-\Psi_{d}\left(k_{n_{d}}^{(d)}\right)\right)} \\
& \cdot \max _{k_{n_{1}<j_{1}<k_{n_{1}+1}^{(1)} \cdots k_{n_{d}}^{(d)}<j_{d}<k_{n_{d}+1}^{(d)}}}\left|\sum_{u_{1}=k_{n_{1}}+1}^{j_{1}} \cdots \sum_{u_{d}=k_{n_{d}+1}^{(d)}}^{j_{d}} S_{\mathbf{u}}\right| \rightarrow 0 \quad(b d)
\end{aligned}
$$

and

$$
\frac{1}{\Psi_{1}\left(n_{1}\right) \cdots \Psi_{d}\left(n_{d}\right)} \sum_{\mathbf{u}<\mathbf{n}} S_{\mathbf{u}} \rightarrow 0 \quad(b d) .
$$

Then relation (1.3) implies relation (1.4), provided

$$
\limsup _{n \rightarrow \infty} \frac{\Psi_{i}\left(k_{n+1}^{(i)}\right)}{\Psi_{i}\left(k_{n}^{(i)}\right)}<\infty, \quad 1 \leq i \leq d,
$$

and relation (1.4) implies relation (1.3), provided

$$
\liminf _{n \rightarrow \infty} \frac{\Psi_{i}\left(k_{n+1}^{(i)}\right)}{\Psi_{i}\left(k_{n}^{(i)}\right)}>1, \quad 1 \leq i \leq d
$$

Consequently, under condition (1.5) and (1.6) the two relations (1.3) and (1.4) are equivalent. 


\section{The main results and proofs}

With the preliminaries accounted for, we can formulate and prove the main results of this paper.

Theorem 1 Let $\left\{X_{\mathbf{n}}, \mathbf{n} \in \mathbb{Z}_{+}^{d}\right\}$ be a random field with centered and integrable random variables being blockwise M-orthogonal with respect to the blocks $\left[2^{n_{1}}, 2^{n_{1}+1}\right) \times\left[2^{n_{2}}, 2^{n_{2}+1}\right) \times$ $\cdots \times\left[2^{n_{d}}, 2^{n_{d}+1}\right)$. Let $\Psi_{i}(\cdot)$ be as in Lemma 2 satisfying (1.5) and (1.6) with $k_{n}^{(i)}=2^{n}, n \in \mathbb{N}$. If, in addition,

$$
\sum_{\mathbf{j} \in \mathbb{Z}_{+}^{d}}\left(\Psi_{1}\left(j_{1}\right) \cdots \Psi_{d}\left(j_{d}\right)\right)^{-2} \cdot\left[\prod_{k=1}^{d}\left(1+\alpha \log _{2} j_{k}\right)^{2}\right] E\left|X_{\mathbf{j}}\right|^{2}<\infty
$$

then

$$
\lim _{\mathbf{n} \rightarrow \infty} \frac{1}{\Psi_{1}\left(n_{1}\right) \cdots \Psi_{d}\left(n_{d}\right)} \sum_{\mathbf{j}<\mathbf{n}} X_{\mathbf{j}}=0 \quad \text { a.s. }
$$

Proof By virtue of Lemma 2, it suffices to show that

$$
\begin{gathered}
\lim _{\mathbf{n} \rightarrow \infty} \frac{1}{\left(\Psi_{1}\left(\left(n_{1}+1\right)^{\alpha}\right)-\Psi_{1}\left(n_{1}^{\alpha}\right)\right) \cdots\left(\Psi_{d}\left(\left(n_{d}+1\right)^{\alpha}\right)-\Psi_{d}\left(n_{d}^{\alpha}\right)\right)} \\
\cdot \max _{n_{1}^{\alpha}<j_{1} \leq\left(n_{1}+1\right)^{\alpha} \ldots n_{d}^{\alpha}<j_{d} \leq\left(n_{d}+1\right)^{\alpha}} \sum_{l_{1}=n_{1}^{\alpha}+1}^{j_{1}} \cdots \sum_{l_{d}=n_{d}^{\alpha}+1}^{j_{d}} X_{\mathbf{1}}=0 \quad \text { a.s. }
\end{gathered}
$$

and

$$
\begin{gathered}
\sup _{\mathbf{n} \succ 1} \mid \frac{1}{\left(\Psi_{1}\left(\left(n_{1}+1\right)^{\alpha}\right)-\Psi_{1}\left(n_{1}^{\alpha}\right)\right) \cdots\left(\Psi_{d}\left(\left(n_{d}+1\right)^{\alpha}\right)-\Psi_{d}\left(n_{d}^{\alpha}\right)\right)} \\
\cdot \max _{\left.n_{1}^{\alpha}<j_{1} \leq\left(n_{1}+1\right)^{\alpha} \ldots n_{d}^{\alpha}<j_{d} \leq n_{d}+1\right)^{\alpha}} \sum_{l_{1}=n_{1}^{\alpha}+1}^{j_{1}} \cdots \sum_{l_{d}=n_{d}^{\alpha}+1}^{j_{d}} X_{\mathbf{l}} \mid<\infty \quad \text { a.s. }
\end{gathered}
$$

At first, we prove (2.1). Applying the Chebyshev's inequality gives

$$
\begin{aligned}
& \sum_{\mathbf{n} \in \mathbb{Z}_{+}^{d}} P\left\{\frac{\max _{n_{1}^{\alpha}<j_{1} \leq\left(n_{1}+1\right)^{\alpha}, \ldots, n_{d}^{\alpha}<j_{d} \leq\left(n_{d}+1\right)^{\alpha}} \sum_{l_{1}=n_{1}^{\alpha}+1}^{j_{1}} \cdots \sum_{l_{d}=n_{d}^{\alpha}+1}^{j_{d}} X_{\mathbf{1}}}{\left(\Psi_{1}\left(\left(n_{1}+1\right)^{\alpha}\right)-\Psi_{1}\left(n_{1}^{\alpha}\right)\right) \cdots\left(\Psi_{d}\left(\left(n_{d}+1\right)^{\alpha}\right)-\Psi_{d}\left(n_{d}^{\alpha}\right)\right)}>\varepsilon\right\} \\
& \leq \frac{1}{\varepsilon^{2}} \sum_{\mathbf{n} \in \mathbb{Z}_{+}^{d}} E\left\{\frac{\max _{n_{1}^{\alpha}<j_{1} \leq\left(n_{1}+1\right)^{\alpha}, \ldots, n_{d}^{\alpha}<j_{d} \leq\left(n_{d}+1\right)^{\alpha} \sum_{l_{1}=n_{1}^{\alpha}+1}^{j_{1}} \cdots \sum_{l_{d}=n_{d}^{\alpha}+1}^{j_{d}} X_{\mathbf{1}}}}{\left(\Psi_{1}\left(\left(n_{1}+1\right)^{\alpha}\right)-\Psi_{1}\left(n_{1}^{\alpha}\right)\right) \cdots\left(\Psi_{d}\left(\left(n_{d}+1\right)^{\alpha}\right)-\Psi_{d}\left(n_{d}^{\alpha}\right)\right)}\right\}^{2} \\
& \leq \frac{\left(c_{1} \cdots c_{d}\right)^{-2}}{\varepsilon^{2}} \sum_{\mathbf{n} \in \mathbb{Z}_{+}^{d}}\left(\Psi_{1}\left(\left(n_{1}+1\right)^{\alpha}\right) \cdots \Psi_{d}\left(\left(n_{d}+1\right)^{\alpha}\right)\right)^{-2} \\
& \cdot E\left\{\max _{n_{1}^{\alpha}<j_{1} \leq\left(n_{1}+1\right)^{\alpha}, \ldots, n_{d}^{\alpha}<j_{d} \leq\left(n_{d}+1\right)^{\alpha}}\left|\sum_{l_{1}=n_{1}^{\alpha}+1}^{j_{1}} \cdots \sum_{l_{d}=n_{d}^{\alpha}+1}^{j_{d}} X_{\mathbf{1}}\right|\right\}^{2},
\end{aligned}
$$


where we used (1.5) and (1.6) yielding $\Psi_{i}\left(\left(n_{i}+1\right)^{\alpha}\right)-\Psi_{i}\left(n_{i}^{\alpha}\right) \geq c_{i} \Psi_{i}\left(\left(n_{i}+1\right)^{\alpha}\right)$ with $c_{i}>0$, $1 \leq i \leq d$, respectively.

Applying Lemma 1 (note that the random variables are within the blocks $M$-orthogonal), we obtain

$$
\begin{aligned}
\frac{\left(c_{1} \cdots c_{d}\right)^{-2}}{\varepsilon^{2}} \sum_{\mathbf{n} \in \mathbb{Z}_{+}^{d}}\left(\Psi_{1}\left(\left(n_{1}+1\right)^{\alpha}\right) \cdots \Psi_{d}\left(\left(n_{d}+1\right)^{\alpha}\right)\right)^{-2} & \left.\cdot E \sum_{n_{1}^{\alpha}<j_{1} \leq\left(n_{1}+1\right)^{\alpha}, \ldots, n_{d}^{\alpha}<j_{d} \leq\left(n_{d}+1\right)^{\alpha}}\left|\sum_{l_{1}=n_{1}^{\alpha}+1}^{j_{1}} \cdots \sum_{l_{d}=n_{d}^{\alpha}+1}^{j_{d}} X_{\mathbf{l}}\right|\right\}^{2} \\
\leq & C \sum_{\mathbf{n} \in \mathbb{Z}_{+}^{d}}\left(\Psi_{1}\left(\left(n_{1}+1\right)^{\alpha}\right) \cdots \Psi_{d}\left(\left(n_{d}+1\right)^{\alpha}\right)\right)^{-2}(m+1)^{d} \\
& \cdot\left(\prod_{i=1}^{d}\left(\log _{2} 2\left(\left(n_{i}+1\right)^{\alpha}-n_{i}^{\alpha}\right)\right)^{2}\right) \\
& \cdot \sum_{l_{1}=n_{1}^{\alpha}+1}^{\left.n_{1}+1\right)^{\alpha}} \ldots \sum_{l_{d}=n_{d}^{\alpha}+1}^{\left(n_{d}+1\right)^{\alpha}} E\left|X_{\mathbf{l}}\right|^{2} \\
\leq & C \sum_{\mathbf{j} \in \mathbb{Z}_{+}^{d}} E\left(\left|X_{\mathbf{j}}\right|^{2}\right) \sum_{n_{1}^{\frac{1}{\alpha}} \cdots j_{1}^{\left(j_{1}+1\right)^{\frac{1}{\alpha}}} \cdots \sum_{j}^{\frac{1}{\alpha}}\left(j_{d}+1\right)^{\frac{1}{\alpha}}}^{n_{d}}\left(\Psi_{1}\left(\left(n_{1}+1\right)^{\alpha}\right) \cdots \Psi_{d}\left(\left(n_{d}+1\right)^{\alpha}\right)\right)^{-2} \\
& \cdot \prod_{k=1}^{d}\left[\log _{2} 2\left(\left(n_{k}+1\right)^{\alpha}-n_{k}^{\alpha}\right)^{2}\right] \\
\leq & C \sum_{\mathbf{j} \in \mathbb{Z}_{+}^{d}}\left(\Psi_{1}\left(j_{1}\right) \ldots \Psi_{d}\left(j_{d}\right)\right)^{-2}\left(\prod_{k=1}^{d}\left(1+\alpha \log _{2} j_{k}\right)^{2}\right) E\left(\left|X_{\mathbf{j}}\right|^{2}\right)<\infty,
\end{aligned}
$$

where $C$ is a constant, which may differ from line to line. From the Borel-Cantelli lemma it follows that

$$
\begin{aligned}
& \lim _{\mathbf{n} \rightarrow \infty} \frac{1}{\left(\Psi_{1}\left(\left(n_{1}+1\right)^{\alpha}\right)-\Psi_{1}\left(n_{1}^{\alpha}\right)\right) \cdots\left(\Psi_{d}\left(\left(n_{d}+1\right)^{\alpha}\right)-\Psi_{d}\left(n_{d}^{\alpha}\right)\right)} \\
& \max _{2^{n_{1}}<j_{1} \leq 2^{n_{1}+1}, \ldots, 2^{n_{d}}<j_{d} \leq 2^{n_{d}+1}} \sum_{l_{1}=2^{n_{1}}+1}^{j_{1}} \ldots \sum_{l_{d}=2^{n_{d}} d}^{j_{d}} X_{\mathbf{I}}=0 \text { a.s. }
\end{aligned}
$$

In order to prove the bounded convergence it remains to show that

$$
\begin{gathered}
\sup _{\mathbf{n} \succ 1} \mid \frac{1}{\left(\Psi_{1}\left(\left(n_{1}+1\right)^{\alpha}\right)-\Psi_{1}\left(n_{1}^{\alpha}\right)\right) \cdots\left(\Psi_{d}\left(\left(n_{d}+1\right)^{\alpha}\right)-\Psi_{d}\left(n_{d}^{\alpha}\right)\right)} \\
\quad \max _{\left.n_{1}^{\alpha}<j_{1} \leq\left(n_{1}+1\right)^{\alpha}, \ldots, n_{d}^{\alpha}<j_{d} \leq n_{d}+1\right)^{\alpha}} \sum_{l_{1}=n_{1}^{\alpha}+1}^{j_{1}} \cdots \sum_{l_{d}=n_{d}^{\alpha}+1}^{j_{d}} X_{\mathbf{1}} \mid<\infty \quad \text { a.s. }
\end{gathered}
$$


Using the same arguments as above with $\varepsilon=1$, we obtain that for almost all $\omega$, there exist only finite many $n_{1}, n_{2}, \ldots, n_{d}$ such that

$$
\begin{gathered}
\mid \frac{1}{\left(\Psi_{1}\left(\left(n_{1}+1\right)^{\alpha}\right)-\Psi_{1}\left(n_{1}^{\alpha}\right)\right) \cdots\left(\Psi_{d}\left(\left(n_{d}+1\right)^{\alpha}\right)-\Psi_{d}\left(n_{d}^{\alpha}\right)\right)} \\
\cdot \max _{n_{1}^{\alpha}<j_{1} \leq\left(n_{1}+1\right)^{\alpha}, \ldots, n_{d}^{\alpha}<j_{d} \leq\left(n_{d}+1\right)^{\alpha}} \sum_{l_{1}=n_{1}^{\alpha}+1}^{j_{1}} \cdots \sum_{l_{d}=n_{d}^{\alpha}+1}^{j_{d}} X_{1} \mid>1 .
\end{gathered}
$$

These complete the proof.

Corollary 1 Let $\left\{X_{\mathbf{n}}, \mathbf{n} \in \mathbb{Z}_{+}^{d}\right\}$ be a random field being blockwise M-orthogonal as in Theorem 1 with centered and integrable random variables. If

$$
\sum_{\mathbf{j} \in \mathbb{Z}_{+}^{d}}\left(j_{1} \cdots j_{d}\right)^{-2} \cdot\left[\prod_{k=1}^{d} \log _{2}\left(2 j_{k}\right)^{2}\right] E\left|X_{\mathbf{j}}\right|^{2}<\infty,
$$

then

$$
\lim _{\mathbf{n} \rightarrow \infty} \frac{1}{n_{1} \cdots n_{d}} \sum_{\mathbf{j}<\mathbf{n}} X_{\mathbf{j}}=0 \quad \text { a.s. }
$$

This follows from Theorem 1 choosing for $\Psi_{i}(\cdot), 1 \leq i \leq d$ the identity function.

In particular, a strong law of large numbers holds for a blockwise $M$-orthogonal random field with bounded $p$ th moment for any $p>1$ a condition, which is just a little bit stronger than the necessary moment condition in the i.i.d. case. Next, choose

$$
\Psi_{i}(t)=t^{\alpha_{i}}, \quad \alpha_{i}>1 / 2,1 \leq i \leq d
$$

in Theorem 1, then we obtain the following corollaries, which are related to the Marcienkiewicz laws in [5].

Corollary 2 Let $\left\{X_{\mathbf{n}}, \mathbf{n} \in \mathbb{Z}_{+}^{d}\right\}$ be a random field as in Theorem 1 . If

$$
\sum_{\mathbf{j} \in \mathbb{Z}_{+}^{d}}\left(j_{1}^{\alpha_{1}} \cdots j_{d}^{\alpha_{d}}\right)^{-2} \cdot\left[\prod_{k=1}^{d} \log _{2}\left(2 j_{k}\right)^{2}\right] E\left|X_{\mathbf{j}}\right|^{2}<\infty,
$$

then

$$
\lim _{\mathbf{n} \rightarrow \infty} \frac{1}{n_{1}^{\alpha_{1}} \cdots n_{d}^{\alpha_{d}}} \sum_{\mathbf{j}<\mathbf{n}} X_{\mathbf{j}}=0 \quad \text { a.s. }
$$

Corollary 3 Let $\left\{X_{\mathbf{n}}, \mathbf{n} \in \mathbb{Z}_{+}^{d}\right\}$ be a random field with blockwise M-orthogonal, centered random variables satisfying $E\left|X_{\mathbf{n}}\right|^{2} \leq M<\infty$ for all $\mathbf{n} \in \mathbb{Z}_{+}^{d}$, then for any $\delta>0$, we have

$$
\lim _{\mathbf{n} \rightarrow \infty} \frac{1}{n_{1}^{\frac{1}{2}}\left(\log n_{1}\right)^{\frac{1}{2}+\delta} \cdots n_{d}^{\frac{1}{2}}\left(\log n_{d}\right)^{\frac{1}{2}+\delta}} \sum_{\mathbf{j}<\mathbf{n}} X_{\mathbf{j}}=0 \quad \text { a.s. }
$$

This follows from our Theorem 1, using $\Psi_{i}(t)=t(\log t)^{\frac{1}{2}+\delta}, 1 \leq i \leq d$. 
Competing interests

The authors declare that they have no competing interests.

\section{Authors' contributions}

WZ and XW carried out the design of the study and performed the analysis, WZ drafted the manuscript. All authors read and approved the final manuscript.

\section{Acknowledgements}

The authors are grateful to the referees for carefully reading the paper and for offering some comments, which helped to improve the paper. The second author is supported by the NNSF of China (No. 11071104), the NSSF of China (13BJY011), PNSF of AnHui (1308085QF113) and GIF of AnHui University of Technology (D2011025).

Received: 6 March 2013 Accepted: 31 July 2013 Published: 14 August 2013

\section{References}

1. Móricz, F, Stadtmüller, U, Thalmaier, SM: Strong laws for blockwise $m$-dependent random fields. J. Theor. Probab. 21, 660-671 (2008)

2. Huan, NV, Quang, NV: The Doob inequality and strong law of large numbers for multidimensional arrays in general Banach spaces. Kybernetika 48(2), 254-267 (2012)

3. Quang, NV, Thanh, LV, Tien, ND: Almost sure convergence for double arrays of block-wise M-dependent random elements in Banach spaces. Georgian Math. J. 18(4), 777-800 (2011)

4. Radchenko, VN: The strong law of large numbers for $m$-orthogonal random variables. Theory Probab. Math. Stat. 33, 111-115 (1985)

5. Gut, A: Marcienkiewicz laws and convergence rates in the law of large numbers for random variables with multidimensional indices. Ann. Probab. 6, 469-482 (1978)

\section{Submit your manuscript to a SpringerOpen ${ }^{\circ}$ journal and benefit from:}

- Convenient online submission

- Rigorous peer review

- Immediate publication on acceptance

- Open access: articles freely available online

- High visibility within the field

- Retaining the copyright to your article 